\title{
Effect of Biochar, Compost and bio-fertilizer on Pea Yield Then, Study its Residual Effect on the Subsequent Pepper Crop
}

\author{
Nahed MM El-Shimi* and Salwa A El-Atbany \\ Vegetables Research Department, Horticulture Research Institute, Agriculture Research Center, Giza, Egypt \\ *Corresponding Author: Nahed MM El-Shimi, Vegetables Research Department, Horticulture Research Institute, Agriculture
}

Research Center, Giza, Egypt.

Received: January 15, 2022; Published: February 17, 2022

DOI: 10.55162/MCAES.02.019

\begin{abstract}
Two field experiments were carried out at two successive seasons of 2018 and 2019 to study the direct effect of biochar, compost and bio-fertilizer ( $\mathrm{N}$ fixing bacteria as Rhizobium leguminosarum for pea or azotobacter for sweet pepper and phosphorus solubilizing microorganisms as phosphoren) under three rates from the recommended mineral fertilizer and their interaction on pea plants (Pisum sativium) cv. Entsar 1 as first experiment and the residual influence of the previous fertilizers on sweet pepper plants (Capsicum annuum L.) cv. California wonder which was planted as second experiment crop. The results according to pea plants gave the best growth attributes, yield and its components were registered by the treatment of biochar with compost and fertilizer. Regarding to the residual influence of biochar, compost and bio-fertilizer led to high values on growth, yield and its characters of sweet pepper fruits in both growing seasons.
\end{abstract}

Keywords: residual effect; biochar; bio-fertilizers; compost; mineral fertilizer

\section{Introduction}

Biochar can steady and hold carbon in agricultural soils for thousands of years, therefore does not apply with each crop, as is usually the case for manures, compost and synthetic fertilizers. Hence, it is clear that biochar can maintain the long term soil fertility [Lehmann et al., (2006)]. Moreover, biochar is a carbon rich solid material, which used as a soil modulation boosts soil fertility by increasing moisture holding capacity, attracting microorganisms and retaining nutrients in soil [Lehmann and Rondon (2006) and Lehmann (2007)].Steiner et al., (2007) reported that, using biochar and organic fertilizers boosts nutrient stocks in the rooting zone, decrease nutrient leaching and improve crop production. On other side Mensah and Frimpong (2018) demonstrated that biochar applied lonely or with compost increased soil quality and improve growth and yield. Meanwhile, biochar integrated with nitrogen fertilization improve sustainability of intensive agriculture by enhancing soil fertility Horák et al., (2020) and Libing Pan et al., (2020).

Organic and bio-fertilizer increase population of microorganisms especially in the root rhizosphere which produce substances encourage plant growth. Concerning the role of organic manures, which integrated with bio-fertilizer Saxena et al., (2013) reported that a bacterial bio-inoculant, Bacillus sp., when inoculated with biochar improve the french beans growth. Saxena et al., (2017) reported that the addition of bio -inoculants to biochar enhancing crop productivity of mung bean.

Pea (Pisum sativum L.) is poor man's meat because it contains high percentage of protein; mineral and periodic carbohydrate content as well as vitamin, iron and zinc (Amarakoon et al., 2012). Furthermore, pea fixes atmospheric nitrogen by contents from microorganisms so it improves soil fertility.

Sweet pepper (Capsicum annuum L.) is an important agricultural crop , not only because of its economic importance but also owing 
to the nutritional value of its fruits since pepper fruits are rich in carbohydrates, proteins, antioxidants, vitamins, minerals, and amino acids, which have beneficial effects regarding the prevention of several disease states (Piñero et al., 2016). Furthermore, pepper fruits contain a high level of vitamin C and other vitamins such as A, E, B1, and B2 (Rodríguez-Ruiz et al., 2017).

This study was aimed to evaluate the influence of the combination among different levels from the recommended mineral fertilizer with biochar, compost as well as bio-fertilizers and their interaction on pea plants and its residual effect as a source of fertilizers for planting sweet pepper.

\section{Material and Methods}

The present work was carried out during two sequential seasons of 2018 and 2019 at the Experimental Farm of Kaha Station, Qalubia Governorate belong to A. R. C. Egypt. The soil was clay in texture with $7.1 \mathrm{pH}, 1.27$ \% organic matters, $110 \mathrm{ppm}$ N, 56ppm P and 98ppm K. This work divided to two experiments to ovulate the influence of adding biochar, compost and bio-fertilizers lonely or in combination between them under three levels from mineral fertilizers for cultivation pea plants (Pisum sativium L) cv. Entsar 1 (first experiment) and notice the residual effect of previous additions on sweet pepper plants (Capsicum annum L) cv. California wonder (second experiment).

In the first planting, each fertilizer was supplied by adding biochar (at rate of 2.5 ton/fed) and commercial compost (El- Waha at 10 ton/fed) which blended with the soil before sowing during soil preparation, while bio-fertilizers (N fixing bacteria as Rhizobium leguminosarum for pea or azotobacter for sweet pepper and phosphorus solubilizing microorganisms as phosphoren at rate of $800 \mathrm{~g} /$ fed.) were mixed to the seeds and seedlings before sowing. While, the mineral fertilizers were added in three levels i.e., $50 \%$, $75 \%$ and $100 \%$ from the recommendation of ministry of agricultural of pea plants i.e., $40 \mathrm{~kg} \mathrm{~N}+30 \mathrm{~kg} \mathrm{P}_{2} \mathrm{O}_{5}+48 \mathrm{~kg} \mathrm{~K} \mathrm{O}_{2}$ fed and pepper plants i.e., $130 \mathrm{~kg} \mathrm{~N}+45 \mathrm{~kg} \mathrm{P}_{2} \mathrm{O}_{5}+72 \mathrm{~kg} \mathrm{~K} \mathrm{O} /$ fed. Which was added after the sowing.

The plot areas were $8.4 \mathrm{~m}^{2}$ and include 3 ridges each of $0.7 \mathrm{~m}$ width and $4.0 \mathrm{~m}$ length. A split - plot design system with three replicates was adopted. This experiment included twenty -four treatments, i.e., the combination among three levels (50\%, $75 \%$ and $100 \%$ control) from the recommended mineral fertilization of pea and sweet pepper were distributed in the main plots and eight treatments which were arranged in the sub plots as follow;

1. Without adding (control).

2. Bio-fertilizer ( $\mathrm{N}$ fixing bacteria as Rhizobium leguminosarum for p0ea or azotobacter for sweet pepper and phosphoren at 800 $\mathrm{g} /$ fed. for both)

3. Commercial compost (El- Waha at 10 ton/fed)

4. Biochare (2.5 ton/fed).

5. Bio-fertilizers + commercial compost.

6. Bio-fertilizers + biochare.

7. Commercial compost + biochare.

8. Commercial compost + biochare + bio-fertilizers .

Chemical and physical properties of the compost and biochare used in this study analyzed in General authority for the agricultural budget fund; Ministry of Agriculture and Land Reclamation are presented in Table 1. 


\begin{tabular}{|l|l|}
\hline Organic fertilizer & Chemical and physical characteristics \\
\hline $\begin{array}{l}\text { Commercial compost } \\
\text { (El-waha compost it is contain } \\
\text { plant waste } 70 \% \text { and animal } \\
\text { waste } 30 \%)\end{array}$ & $1.3 \% \mathrm{~N}, 0.8 \mathrm{P} \%, 0.7 \% \mathrm{~K}, \mathrm{EC} 6.2, \mathrm{pH} 6.8$ matter, 37\%Organic C/N ratio 01:18, 25\% moisture \\
\hline $\begin{array}{l}\text { Biochar } \\
\text { Produced from residual trees } \\
\text { wood. }\end{array}$ & $\begin{array}{l}\text { Moisture content 3.5\%, Ash content 3.3\%, EC1.3 dS m-1, pH 7.6, Total organic carbon\%94, C:N } \\
\text { Ratio 83.9, Total Nitrogen } \% 1.12, \text { Total Phosphorus \%0.106,Total Potassium } 2.9 \%, \text { Magnesium } \\
0.36 \%, \text { Calcium 1.1\%, Cation exchangeable capacity mmolc 16kg-1 }\end{array}$ \\
\hline
\end{tabular}

Table 1: Chemical and physical properties of the organic fertilizers.

Seeds of pea were sown on $21^{\text {th }}$ and $19^{\text {th }}$ November in the first and the second seasons, respectively in hills on one side of the ridges at $5-7 \mathrm{~cm}$. While sweet pepper seeds (second experiment) were sown under plastic house in nursery at the first week of February during both seasons and received natural agricultural practices. After 50 days from sowing healthy seedlings were selected and transplanted in the field plots at $35 \mathrm{~cm}$ apart between the seedlings in one side of the ridge after harvesting pea.

The other agricultural practices for pea and sweet pepper were carried out as the recommendation of ministry of agricultural.

\section{Recoded Data}

First experiment

Vegetative growth parameters

Three plants were randomly chosen from the each replicate from treatment at flowering stage and beginning of fruiting stage at 60 days after sowing to determine the following:

- $\quad$ Plant length, stem diameter, number of brunches and leaves/ plant and dry weight (g/plant).

- Leaf area was calculated at flowering stage and beginning of fruiting stage (at 72 days after sowing) from the fourth upper leaves according to the following formula of Wallace and Munger (1965).

$$
\text { Leaf area }\left(\mathrm{cm}^{2}\right)=\frac{\text { Leaves dry weight }(\mathrm{gm}) \mathrm{xdisk} \text { area }\left(\mathrm{cm}^{2}\right)}{\text { Disk dry weight }(\mathrm{gm})}
$$

\section{Yield component}

A random sample of 10 fresh pods (in green mature stage at edible stage) from each plot at the second picking was taken to determine pod length $(\mathrm{cm})$, pod diameter $(\mathrm{cm})$, average of fresh pod weight $(\mathrm{g})$, number of seeds/pod, fresh and dry weight of 100 seeds (g) and total pod yield (ton/fed.)

\section{Second experiment}

Vegetative growth parameters

Three plants were randomly chosen from the three replicates at the flowering stage at 60 days after transplanting to determine the following data.

- $\quad$ Plant length (main stem $\mathrm{cm}$ ), stem diameter $(\mathrm{cm})$ - number of brunches/ plant and leaves number/plant, dry weight $(\mathrm{g} / \mathrm{plant}$

- The leaf area was calculated at flowering stage (after 60 days from transplanting) from the fourth upper leaves according to the following formula of Wallace and Munger (1965).

\section{Physical characters of fruit yield}

- $\quad$ Fruit weight (g), early fruit yield (ton/fed) as the first and second pickings and total yield (ton/fed) were recorded.

- $\quad$ Fruits physical characters: Five sweet pepper fruits were randomly selected from each replicate at the second picking 95 days 
after transplanting to determine; fruit length $(\mathrm{cm})$, fruit diameter $(\mathrm{cm})$, fruit flesh thickness $(\mathrm{cm})$ and loculi number.

\section{The chemical properties}

- Total leaf chlorophyll reading was measured using Minolta chlorophyll meter SPAD- 501 as SPAD units at flowering stage and beginning of fruiting stage at 72 days after sowing from the fourth upper leaves.

- $\quad$-Ascorbic acid: (Vitamin C mg/100g fresh weight) using the die 2, 6 dichlorophenol indophenols, method as described by Ranganna (1979).

\section{Statistical analysis}

Data were subjected to the proper analysis of variance (split plot design) as described by Snedecor and Cochran (1980) using M. stat program. Averages between treatments were differentiated by using LSD at $5 \%$ level.

\section{Results and Discussion}

\section{First experiment}

Parameters of vegetative growth

Effect of mineral fertilizer levels

Data registered in Table (2) showed the best growth attributes, i.e., plant length, leaf area and dry weight of plant with adding level of $100 \%$ from the recommended mineral fertilization in both growing season except, plant dry weight with fertilized $50 \%$ followed by $100 \%$ of the recommended mineral fertilization in the first season, while leaves number was the best under using level of $75 \%$ from the recommended rate of NPK. Mineral fertilizers increase growth attributes of pea plants (Mishra et al., 2010). Chemical fertilizers could improve plant growth parameters to the role of nitrogen in nucleic acids and protein synthesis, and phosphorus as an essential component of the energy compounds and phosphoprotein, also potassium plays as an activator of many enzymes (Helgi and Rolfe, 2005). The same trend was obtained by Jayasinghe and Weerawansha (2018).

\section{Effect of biochar, compost and bio-fertilizer treatments}

Results recorded in Table (2) clear that, all studied growth attributes were increased significantly by all treatments. From this concern biochar applied lonely or with compost and bio fertilizer (Rhizobium and phosphorene) increased plant length in the two seasons under study also enhance leaves and branches number, plant dry weight and leaf area in both growing seasons. These result may be due to the ability of biochar to increase maintain retention nutrient especially nitrogen content in the soil as mentioned by (Lehmann et al., 2003; Steiner et al., 2008). Moreover, biochar is a carbon rich solid material, which improves soil fertility by increasing moisture holding capacity, attracting beneficial microorganisms, improving cation exchange capacity, as well as retaining nutrients in soil [Lehmann and Rondon (2006) and Lehmann (2007). These results agreement with those obtained by Ganie et al., (2010) on pea, Tagore et al., (2013) on chickpea Feleafel and Mirdad (2014) on snap bean. Also, Mensah and Frimpong (2018) found that biochar and compost applied alone or together increased significantly total organic carbon, available phosphorus, and mineral nitrogen whereas reduced exchangeable acidity. Additionally, they increased plant height and stem girth and dry matter. Therefore, this study cleared that biochar applied alone or with compost offers enhance soil quality and improve crop yield. 
Effect of Biochar, Compost and bio-fertilizer on Pea Yield Then, Study its Residual Effect on the Subsequent Pepper Crop

\begin{tabular}{|c|c|c|c|c|c|c|c|c|c|c|}
\hline \multirow[t]{2}{*}{ Treatments } & \multicolumn{2}{|c|}{$\begin{array}{c}\text { Plant } \\
\text { length }(\mathrm{cm}) \\
\end{array}$} & \multicolumn{2}{|c|}{$\begin{array}{l}\text { No. of leaves/ } \\
\text { Plant }\end{array}$} & \multicolumn{2}{|c|}{$\begin{array}{c}\text { No. of. Brunches/ } \\
\text { plant }\end{array}$} & \multicolumn{2}{|c|}{$\begin{array}{c}\text { Dry weight /plant } \\
\text { (g) }\end{array}$} & \multicolumn{2}{|c|}{ Leaf area $\left(\mathrm{cm}^{2}\right)$} \\
\hline & 2018 & 2019 & 2018 & 2019 & 2018 & 2019 & 2018 & 2019 & 2018 & 2019 \\
\hline \multicolumn{11}{|c|}{ Fertilization levels } \\
\hline $100 \%$ (cont) & 45.78 & 44.02 & 13.08 & 12.37 & 1.75 & 1.48 & 3.25 & 3.03 & 218.51 & 200.52 \\
\hline $75 \%$ & 45.08 & 43.04 & 15.34 & 14.39 & 1.75 & 1.52 & 3.03 & 2.81 & 209.63 & 192.69 \\
\hline $50 \%$ & 43.24 & 42.22 & 14.84 & 13.88 & 2.19 & 1.90 & 3.27 & 2.99 & 203.45 & 188.79 \\
\hline L.S.D at $5 \%$ level & 0.34 & 1.28 & 0.68 & 0.11 & 0.07 & 0.11 & 0.03 & 0.12 & 7.58 & 4.99 \\
\hline \multicolumn{11}{|c|}{ Soil adding } \\
\hline Control & 39.90 & 40.07 & 12.43 & 11.91 & 1.33 & 1.08 & 2.45 & 2.27 & 164.19 & 151.12 \\
\hline Bio-fertilizer & 46.25 & 44.63 & 14.17 & 13.38 & 1.67 & 1.50 & 2.78 & 2.51 & 183.61 & 170.75 \\
\hline Compost & 46.91 & 44.18 & 13.60 & 12.97 & 1.77 & 1.50 & 2.90 & 2.70 & 225.10 & 199.02 \\
\hline Biochar & 47.58 & 45.67 & 15.38 & 14.23 & 2.17 & 1.88 & 3.79 & 3.32 & 226.41 & 207.93 \\
\hline Bio-fertilizer+ compost & 41.50 & 40.22 & 14.43 & 13.48 & 2.10 & 1.88 & 3.33 & 3.30 & 215.99 & 201.97 \\
\hline Bio-fertilizer+ biochar & 42.10 & 41.30 & 15.33 & 14.17 & 1.83 & 1.58 & 2.72 & 2.56 & 230.30 & 215.25 \\
\hline Compost+ biochar & 47.33 & 44.67 & 14.50 & 13.79 & 1.83 & 1.54 & 3.63 & 3.27 & 209.17 & 188.74 \\
\hline Bio-fertilizer+Compost+ biochar & 46.00 & 44.01 & 15.50 & 14.63 & 2.46 & 2.08 & 3.83 & 3.61 & 229.45 & 218.53 \\
\hline L.S.D at $5 \%$ level & 0.82 & 0.81 & 0.73 & 0.71 & 0.09 & 0.09 & 0.08 & 0.09 & 6.75 & 11.54 \\
\hline
\end{tabular}

Table 2: The direct effect of mineral fertilizer levels, biochar, bio-fertilizer, compost, on vegetative growth of pea (Pisum sativium) during the two seasons of 2018 and 2019.

\section{Effect of the interaction}

Data about the parameters of pea plants growth cleared in Table (3). These parameters were differing regarding to the treatments under investigation, however adding bio fertilizer with 75\% from recommended NPK gave tallest plants. The highest leaves number / plant were recorded by adding biochar alone or bio fertilizer combined with compost or biochar under $50 \%$ from recommended NPK. Meanwhile, added 50\% from recommended NPK was significant to increase the number of branches / plant when added bio fertilizer with compost and increased obtained the augment results of dry weight when the soil treated with biochar, compost and bio fertilizers together. While $100 \%$ of the recommended mineral fertilization and biochar combined with bio fertilizer led to produce plants characterize with width leaf area, these results were true in both growing seasons. This may be to biochar with nitrogen fertilization appears to be promising practice to improve sustainability of intensive agriculture by enhancing chemical properties of soil Horák et al., (2020) and Libing Pan et al., (2020). Also, using bio-fertilizers beside addition chemical fertilizer with small amount has been encouraging the growth vigor peas. Since, the bio-fertilizers are safe, low cost and easy in application Byan and EL-Shimi (2014). 
Effect of Biochar, Compost and bio-fertilizer on Pea Yield Then, Study its Residual Effect on the Subsequent Pepper Crop

\begin{tabular}{|c|c|c|c|c|c|c|c|c|c|c|c|}
\hline \multirow{2}{*}{\multicolumn{2}{|c|}{ Treatments }} & \multicolumn{2}{|c|}{$\begin{array}{l}\text { Plant length } \\
\text { (cm) }\end{array}$} & \multicolumn{2}{|c|}{$\begin{array}{c}\text { No. of leaves/ } \\
\text { plant }\end{array}$} & \multicolumn{2}{|c|}{$\begin{array}{c}\text { No. of. Brunches/ } \\
\text { plant }\end{array}$} & \multicolumn{2}{|c|}{$\begin{array}{c}\text { Dry weight / } \\
\text { plant(g) }\end{array}$} & \multicolumn{2}{|c|}{ Leaf area $\left(\mathrm{cm}^{2}\right)$} \\
\hline & & 2018 & 2019 & 2018 & 2019 & 2018 & 2019 & 2018 & 2019 & 2018 & 2019 \\
\hline \multirow{8}{*}{ 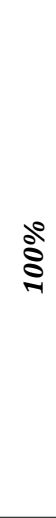 } & Control & 37.85 & 39.70 & 11.30 & 10.73 & 1.50 & 1.00 & 2.85 & 2.60 & 168.06 & 166.02 \\
\hline & Bio-fertilizer & 44.00 & 43.50 & 14.00 & 13.00 & 2.00 & 1.75 & 3.10 & 2.78 & 193.02 & 179.99 \\
\hline & Compost & 48.30 & 45.30 & 12.30 & 11.90 & 1.30 & 1.25 & 3.20 & 3.10 & 253.70 & 198.85 \\
\hline & Biochar & 48.25 & 46.5 & 14.00 & 13.12 & 1.50 & 1.25 & 3.75 & 3.27 & 264.39 & 229.77 \\
\hline & $\begin{array}{l}\text { Bio-fertilizer+com- } \\
\text { post }\end{array}$ & 47.00 & 44.65 & 12.00 & 11.85 & 1.30 & 1.30 & 3.25 & 3.20 & 195.69 & 194.36 \\
\hline & $\begin{array}{l}\text { Bio-fertilizer+ bio- } \\
\text { char }\end{array}$ & 45.30 & 43.65 & 14.50 & 13.00 & 2.00 & 1.75 & 2.70 & 2.65 & 267.30 & 237.02 \\
\hline & Compost+ biochar & 47.50 & 44.00 & 12.00 & 11.75 & 1.50 & 1.25 & 3.55 & 3.15 & 202.97 & 195.28 \\
\hline & $\begin{array}{l}\text { Bio-fertilizer+ Com- } \\
\text { post+ biochar }\end{array}$ & 48.00 & 44.84 & 14.50 & 13.63 & 2.88 & 2.25 & 3.60 & 3.45 & 202.97 & 206.84 \\
\hline \multirow{8}{*}{ ํํ } & Control & 41.10 & 40.50 & 14.50 & 14.00 & 1.50 & 1.25 & 2.30 & 2.20 & 165.25 & 155.88 \\
\hline & Bio-fertilizer & 49.75 & 47.50 & 14.50 & 13.63 & 1.50 & 1.38 & 2.5 & 2.40 & 188.94 & 169.01 \\
\hline & Compost & 47.75 & 43.88 & 16.50 & 15.25 & 2.00 & 1.75 & 2.95 & 2.68 & 216.07 & 207.65 \\
\hline & Biochar & 48.50 & 45.50 & 16.50 & 15.25 & 2.50 & 2.13 & 3.60 & 3.10 & 202.50 & 190.85 \\
\hline & $\begin{array}{l}\text { Bio-fertilizer+ com- } \\
\text { post }\end{array}$ & 40.50 & 39.25 & 14.75 & 13.50 & 1.50 & 1.25 & 2.90 & 2.90 & 216.38 & 195.51 \\
\hline & $\begin{array}{l}\text { Bio-fertilizer+ bio- } \\
\text { char }\end{array}$ & 42.50 & 42.25 & 15.00 & 14.00 & 1.50 & 1.25 & 2.60 & 2.50 & 211.80 & 210.98 \\
\hline & Compost+ biochar & 47.50 & 43.25 & 15.00 & 14.50 & 1.50 & 1.38 & 3.50 & 3.10 & 229.07 & 187.58 \\
\hline & $\begin{array}{l}\text { Bio-fertilizer+ Com- } \\
\text { post+ biochar }\end{array}$ & 43.00 & 42.18 & 16.00 & 15.00 & 2.00 & 1.75 & 3.80 & 3.50 & 247.00 & 224.05 \\
\hline \multirow{8}{*}{ Бे } & Control & 40.75 & 40.00 & 11.50 & 11.00 & 1.00 & 1.00 & 2.20 & 2.00 & 159.26 & 131.47 \\
\hline & Bio-fertilizer & 45.00 & 42.88 & 14.00 & 13.50 & 1.50 & 1.38 & 2.70 & 2.35 & 168.88 & 163.26 \\
\hline & Compost & 44.68 & 43.35 & 12.00 & 11.75 & 2.00 & 1.50 & 2.55 & 2.33 & 205.53 & 190.55 \\
\hline & Biochar & 46.00 & 45.00 & 15.63 & 14.32 & 2.50 & 2.25 & 4.03 & 3.60 & 212.34 & 203.18 \\
\hline & $\begin{array}{l}\text { Bio-fertilizer+com- } \\
\text { post }\end{array}$ & 37.00 & 36.75 & 16.55 & 15.10 & 3.50 & 3.10 & 3.85 & 3.80 & 235.90 & 216.05 \\
\hline & $\begin{array}{l}\text { Bio-fertilizer+ bio- } \\
\text { char }\end{array}$ & 38.50 & 38.00 & 16.50 & 14.75 & 2.00 & 1.75 & 2.8 & 2.53 & 211.80 & 197.76 \\
\hline & Compost+ biochar & 47.00 & 46.75 & 16.50 & 15.13 & 2.50 & 2.00 & 3.85 & 3.49 & 195.46 & 183.35 \\
\hline & $\begin{array}{l}\text { Bio-fertilizer+ Com- } \\
\text { post+ biochar }\end{array}$ & 47.00 & 45.00 & 16.00 & 15.50 & 2.50 & 2.25 & 4.09 & 3.88 & 238.39 & 224.69 \\
\hline \multicolumn{2}{|c|}{ L.S.D at $5 \%$ level } & 0.50 & 0.49 & 0.44 & 0.43 & 0.05 & 0.05 & 0.05 & 0.06 & 4.09 & 7.00 \\
\hline
\end{tabular}

Table 3: The direct effect of the interaction between mineral fertilizer levels, biochar, bio-fertilizers, compost, on vegetative growth of pea (Pisum sativium) during the two seasons of 2018 and 2019.

\section{Yield component}

\section{Effect of mineral fertilizer levels}

Data in Table (4) revealed that addition $50 \%$ of the recommended mineral fertilization increased significantly pod length, dry weight of 100 seeds as well as total yield $t /$ fed in both growing seasons. While the treatment of full recommended mineral NPK dose increased the fresh weight of both pod and 100 seeds. On the other side, there no significant differences between treatments were detection 
Effect of Biochar, Compost and bio-fertilizer on Pea Yield Then, Study its Residual Effect on the Subsequent Pepper Crop

pod diameter and seeds number/pod in both growing seasons. Chemical fertilizers could promote plant growth which that reflects on yield and its components. Whereas, nitrogen enters installation nucleic acids and protein synthesis, and phosphorus as an essential component of the energy compounds and phosphoprotein, also potassium plays as stimulating of enzymes (Helgi and Rolfe, 2005).

\begin{tabular}{|c|c|c|c|c|c|c|c|c|c|c|c|c|c|c|}
\hline \multirow[t]{2}{*}{ Treatments } & \multicolumn{2}{|c|}{$\begin{array}{l}\text { Pod length } \\
\quad(\mathrm{cm})\end{array}$} & \multicolumn{2}{|c|}{$\begin{array}{c}\text { Pod diameter } \\
\text { (cm) }\end{array}$} & \multicolumn{2}{|c|}{$\begin{array}{l}\text { Pod fresh } \\
\text { weight }(g)\end{array}$} & \multicolumn{2}{|c|}{$\begin{array}{c}\text { Seed num- } \\
\text { ber/pod }\end{array}$} & \multicolumn{2}{|c|}{$\begin{array}{c}\text { Fresh weight } \\
\text { of } 100 \\
\text { seeds }(g)\end{array}$} & \multicolumn{2}{|c|}{$\begin{array}{l}\text { Dry weight of } \\
100 \text { seeds }(g)\end{array}$} & \multicolumn{2}{|c|}{$\begin{array}{c}\text { Total yield } \\
\text { t/fed }\end{array}$} \\
\hline & 2018 & 2019 & 2018 & 2019 & 2018 & 2019 & 2018 & 2019 & 2018 & 2019 & 2018 & 2019 & 2018 & 2019 \\
\hline \multicolumn{15}{|c|}{ Fertilizer rates } \\
\hline $100 \%$ (cont) & 11.75 & 11.40 & 1.43 & 1.51 & 11.26 & 10.73 & 8.50 & 8.31 & 64.00 & 62.51 & 13.77 & 13.04 & 3.49 & 3.02 \\
\hline $75 \%$ & 11.94 & 11.63 & 1.46 & 1.47 & 10.84 & 10.64 & 8.55 & 8.34 & 61.10 & 60.32 & 13.93 & 13.38 & 2.90 & 2.90 \\
\hline $50 \%$ & 12.02 & 11.91 & 1.48 & 1.45 & 10.92 & 10.57 & 8.38 & 8.21 & 63.21 & 61.71 & 14.29 & 13.75 & 3.70 & 3.40 \\
\hline $\begin{array}{l}\text { L.S.D at } 5 \% \\
\text { level }\end{array}$ & 0.01 & 0.03 & N.S & N.S & 0.22 & 0.12 & N.S & N.S & 0.38 & 0.34 & 0.16 & 0.17 & 0.03 & 0.11 \\
\hline \multicolumn{15}{|c|}{ Soil adding } \\
\hline Control & 11.03 & 10.88 & 1.43 & 1.41 & 10.07 & 9.45 & 7.68 & 7.47 & 58.10 & 57.00 & 11.37 & 10.73 & 2.16 & 1.90 \\
\hline Bio-fertilizer & 11.90 & 11.58 & 1.45 & 1.49 & 10.83 & 10.19 & 8.20 & 8.04 & 64.14 & 62.83 & 13.94 & 13.51 & 3.05 & 2.73 \\
\hline Compost & 12.13 & 11.63 & 1.49 & 1.49 & 11.26 & 10.89 & 8.63 & 8.28 & 63.63 & 61.82 & 13.72 & 12.82 & 3.29 & 3.11 \\
\hline Biochar & 12.11 & 11.84 & 1.48 & 1.50 & 11.03 & 10.83 & 8.53 & 8.45 & 63.67 & 62.24 & 14.29 & 13.61 & 3.87 & 3.57 \\
\hline $\begin{array}{l}\text { Bio-fertilizer+ } \\
\text { compost }\end{array}$ & 12.01 & 11.53 & 1.44 & 1.47 & 11.36 & 10.85 & 8.60 & 8.47 & 60.87 & 60.07 & 13.80 & 13.11 & 3.27 & 3.18 \\
\hline $\begin{array}{l}\text { Bio-fertilizer+ } \\
\text { biochar }\end{array}$ & 12.10 & 11.62 & 1.44 & 1.49 & 10.92 & 10.80 & 8.78 & 8.52 & 64.35 & 63.10 & 14.48 & 13.73 & 4.03 & 3.54 \\
\hline $\begin{array}{l}\text { Compost+ } \\
\text { biochar }\end{array}$ & 12.10 & 11.80 & 1.48 & 1.50 & 11.44 & 11.27 & 8.60 & 8.44 & 63.33 & 62.48 & 14.48 & 14.18 & 3.31 & 3.17 \\
\hline $\begin{array}{l}\text { Bio-fertilizer+ } \\
\text { Compost+ } \\
\text { biochar }\end{array}$ & 12.23 & 11.91 & 1.45 & 1.48 & 11.15 & 10.87 & 8.77 & 8.62 & 64.07 & 62.57 & 15.90 & 15.43 & 3.93 & 3.65 \\
\hline $\begin{array}{l}\text { L.S.D at } 5 \% \\
\text { level }\end{array}$ & 0.08 & 0.07 & N.S & N.S & 0.24 & 0.28 & 0.29 & 0.19 & 0.24 & 0.30 & 0.21 & 0.19 & 0.09 & 0.08 \\
\hline
\end{tabular}

Table 4: The direct effect of mineral fertilizer levels, biochar, bio-fertilizer, compost, on yield and its components of pea (Pisum sativium) during the two seasons of 2018 and 2019.

\section{Effect of biochar, compost and bio-fertilizer treatments}

As secured in Table (4) all treatments significantly increased total yield of pea and its components compared to the control. Adding biochar only increased pod length. Whereas adding biochar lonely or with compost and bio fertilizer increased pod length, pod fresh weight, seeds number in the pods, the fresh and dry weight of 100 seeds as well as total yield t/fed in both growing seasons. Regarding to pod diameter, all treatments have no significant effect in both growing seasons. These results are agreement with data in Table (2) in this study. This was due to using organic and bio-fertilizer encourage population of microorganisms t which produce stimulate plant growth. Also addition of bio -inoculants to biochar is useful practice for increasing productivity Saxena et al., (2017). This is consistent with that found by Glaser and Birk (2012), Liu et al., (2012), Schulz and Glaser (2012), Fischer and Glaser (2012) and Trupiano et al., (2017) on lettuce.

\section{Effect of the interaction}

Results in Table (5) registered that, the best values for each of pod length, fresh weight of 100 seeds and the yield ton/fed were ob- 
tained in the plots fertilized by adding 50\% of recommended mineral NPK by adding biochar with bio fertilizer. Meanwhile, pod fresh weight increased positively by adding $100 \%$ of recommended mineral NPK and adding biochar with compost. While, adding biochar with compost and bio fertilizer led to increase number of seeds / pod with $75 \%$ of recommended mineral NPK as well as dry weight of 100 seeds with $50 \%$ of recommended mineral NPK. Regarding to pod diameter, there was no significant effect among all treatments. These results were true in the both seasons and agreement with Zaghloul et al., (2015) on pea which proved that using of bio-fertilization which reduced the amount used from chemical fertilizer by $50 \%$.

\begin{tabular}{|c|c|c|c|c|c|c|c|c|c|c|c|c|c|c|c|}
\hline \multicolumn{2}{|c|}{ Treatments } & \multicolumn{2}{|c|}{$\begin{array}{l}\text { Pod length } \\
\text { (cm) }\end{array}$} & \multicolumn{2}{|c|}{$\begin{array}{c}\text { Pod diameter } \\
\text { (cm) }\end{array}$} & \multicolumn{2}{|c|}{$\begin{array}{l}\text { Pod fresh } \\
\text { weight (g) }\end{array}$} & \multicolumn{2}{|c|}{$\begin{array}{c}\text { Seed num- } \\
\text { ber/pod }\end{array}$} & \multicolumn{2}{|c|}{$\begin{array}{c}\text { Fresh weight of } \\
100 \text { seeds (g) }\end{array}$} & \multicolumn{2}{|c|}{$\begin{array}{l}\text { Dry weight of } \\
100 \text { seeds }(g)\end{array}$} & \multicolumn{2}{|c|}{$\begin{array}{c}\text { Total yield } \\
\text { t/fed }\end{array}$} \\
\hline & & 2018 & 2019 & 2018 & 2019 & 2018 & 2019 & 2018 & 2019 & 2018 & 2019 & 2018 & 2019 & 2018 & 2019 \\
\hline \multirow{8}{*}{$\frac{1}{8}$} & Control & 11.19 & 10.90 & 1.44 & 1.45 & 10.74 & 9.78 & 7.80 & 7.70 & 58.50 & 57.50 & 11.90 & 11.00 & 2.48 & 2.02 \\
\hline & $\begin{array}{l}\text { Bio-fertil- } \\
\text { izer }\end{array}$ & 11.38 & 11.20 & 1.40 & 1.57 & 11.03 & 9.71 & 8.00 & 7.80 & 65.70 & 64.55 & 13.90 & 13.35 & 3.04 & 2.09 \\
\hline & Compost & 12.10 & 11.03 & 1.46 & 1.54 & 11.90 & 11.44 & 8.60 & 8.20 & 65.70 & 64.65 & 12.80 & 12.20 & 3.71 & 3.40 \\
\hline & Biochar & 11.95 & 11.80 & 1.50 & 1.52 & 11.10 & 10.74 & 8.50 & 8.45 & 65.70 & 62.50 & 14.03 & 13.06 & 4.11 & 3.39 \\
\hline & $\begin{array}{l}\text { Bio-fer- } \\
\text { tilizert } \\
\text { compost }\end{array}$ & 11.85 & 11.55 & 1.37 & 1.53 & 11.57 & 10.79 & 8.70 & 8.50 & 64.10 & 62.50 & 13.90 & 13.35 & 3.56 & 3.38 \\
\hline & $\begin{array}{l}\text { Bio-fertiliz- } \\
\text { er+biochar }\end{array}$ & 11.60 & 11.35 & 1.38 & 1.51 & 10.64 & 10.67 & 8.90 & 8.65 & 63.50 & 62.18 & 14.40 & 13.50 & 3.73 & 3.15 \\
\hline & $\begin{array}{l}\text { Compost+ } \\
\text { biochar }\end{array}$ & 12.10 & 11.90 & 1.48 & 1.49 & 12.05 & 11.90 & 8.80 & 8.65 & 64.60 & 64.23 & 14.50 & 14.05 & 3.22 & 3.10 \\
\hline & $\begin{array}{l}\text { Bio-fer- } \\
\text { tilizer+ } \\
\text { Compost+ } \\
\text { biochar }\end{array}$ & 11.85 & 11.45 & 1.43 & 1.47 & 11.06 & 10.79 & 8.70 & 8.50 & 64.20 & 62.00 & 14.70 & 13.80 & 4.08 & 3.64 \\
\hline \multirow{8}{*}{ ì } & Control & 11.10 & 10.80 & 1.42 & 1.39 & 9.90 & 9.47 & 7.73 & 7.60 & 58.60 & 57.10 & 11.60 & 11.10 & 2.16 & 1.90 \\
\hline & $\begin{array}{l}\text { Bio-fertil- } \\
\text { izer }\end{array}$ & 12.20 & 12.15 & 1.47 & 1.44 & 10.53 & 10.41 & 8.10 & 8.05 & 63.97 & 62.50 & 13.57 & 12.88 & 2.83 & 2.95 \\
\hline & Compost & 12.20 & 12.10 & 1.50 & 1.47 & 10.74 & 10.64 & 8.90 & 8.45 & 62.00 & 60.90 & 14.10 & 13.05 & 2.63 & 2.82 \\
\hline & Biochar & 12.33 & 12.15 & 1.48 & 1.50 & 11.14 & 10.96 & 8.50 & 8.40 & 59.40 & 59.00 & 13.90 & 13.43 & 3.23 & 3.15 \\
\hline & $\begin{array}{l}\text { Bio-fer- } \\
\text { tilizert } \\
\text { compost }\end{array}$ & 11.88 & 11.13 & 1.46 & 1.47 & 11.17 & 10.94 & 8.30 & 8.30 & 59.60 & 59.15 & 13.85 & 12.98 & 3.22 & 3.09 \\
\hline & $\begin{array}{l}\text { Bio-fertiliz- } \\
\text { er+biochar }\end{array}$ & 11.95 & 11.13 & 1.43 & 1.48 & 10.73 & 10.62 & 8.93 & 8.56 & 62.35 & 61.60 & 14.20 & 13.80 & 3.08 & 3.11 \\
\hline & $\begin{array}{l}\text { Compost+ } \\
\text { biochar }\end{array}$ & 12.26 & 11.63 & 1.48 & 1.50 & 11.38 & 11.21 & 8.90 & 8.56 & 61.00 & 60.70 & 14.00 & 13.73 & 3.13 & 3.19 \\
\hline & $\begin{array}{l}\text { Bio-fer- } \\
\text { tilizer+ } \\
\text { Compost+ } \\
\text { biochar }\end{array}$ & 12.30 & 11.93 & 1.47 & 1.51 & 11.15 & 10.87 & 9.00 & 8.80 & 61.90 & 61.60 & 16.25 & 16.10 & 2.89 & 2.97 \\
\hline
\end{tabular}


Effect of Biochar, Compost and bio-fertilizer on Pea Yield Then, Study its Residual Effect on the Subsequent Pepper Crop

\begin{tabular}{|l|l|c|c|c|c|c|c|c|c|c|c|c|c|c|}
\hline & 10.80 & 10.95 & 1.43 & 1.39 & 9.57 & 9.11 & 7.50 & 7.10 & 57.20 & 56.40 & 10.60 & 10.10 & 1.86 & 1.78 \\
\hline $\begin{array}{l}\text { Control } \\
\text { Bio-fertil- }\end{array}$ & 12.13 & 11.38 & 1.49 & 1.46 & 10.92 & 10.46 & 8.50 & 8.28 & 62.75 & 61.45 & 14.35 & 14.30 & 3.27 & 3.16 \\
\hline Compost & 12.10 & 11.00 & 1.53 & 1.45 & 11.15 & 10.58 & 8.40 & 8.20 & 63.20 & 59.90 & 14.27 & 13.20 & 3.53 & 3.11 \\
\hline Biochar & 12.05 & 11.58 & 1.47 & 1.48 & 10.85 & 10.80 & 8.60 & 8.50 & 65.90 & 65.23 & 14.95 & 14.33 & 4.29 & 4.17 \\
\hline $\begin{array}{l}\text { Bio-fer- } \\
\text { tilizer+ } \\
\text { compost }\end{array}$ & 12.30 & 11.90 & 1.48 & 1.40 & 11.34 & 10.82 & 8.80 & 8.60 & 58.90 & 58.55 & 13.65 & 13.00 & 3.03 & 3.07 \\
\hline $\begin{array}{l}\text { Bio-fertiliz- } \\
\text { er+ biochar }\end{array}$ & 12.55 & 12.37 & 1.52 & 1.49 & 11.40 & 11.11 & 8.50 & 8.35 & 67.20 & 65.53 & 14.85 & 13.90 & 5.28 & 4.36 \\
\hline $\begin{array}{l}\text { Compost+ } \\
\text { biochar }\end{array}$ & 11.95 & 11.88 & 1.48 & 1.51 & 10.88 & 10.70 & 8.10 & 8.10 & 64.40 & 62.50 & 14.93 & 14.78 & 3.57 & 3.21 \\
\hline $\begin{array}{l}\text { Bio-fer- } \\
\text { tilizer+ } \\
\text { Compost+ } \\
\text { biochar }\end{array}$ & 12.55 & 12.35 & 1.47 & 1.46 & 11.24 & 10.94 & 8.60 & 8.55 & 66.10 & 64.10 & 16.75 & 16.38 & 4.81 & 4.36 \\
\hline L.S.D at 5 \% level
\end{tabular}

Table 5: The direct effect of the interaction between mineral fertilizer levels, biochar, bio-fertilizers, compost, on pea (Pisum sativium) yield and its components during the two seasons of 2018 and 2019.

Second experiment (sweet pepper plantation)

Vegetative

Effect of mineral fertilizer levels

Sweet pepper growth attributes, which planting in the same place of the previous pea plantation are secured in Table (6). It noticed that, highest rates of the recommended mineral fertilizer of (NPK) 100\% followed by $75 \%$ gave significant increase on the growth parameters as well as chlorophyll leaf concentration of plant except plant length and stem diameter in both growing seasons and number of leaves/plant in the second season. Similar results was found) in sweet pepper by Toungous (2017) who indicated that increasing NPK-fertilizers encourage growth. Also Gaafar et al., (2019) and El-Atbany and Byan (2019) found that, adding $100 \%$ followed by $75 \%$ of the recommended doses of mineral fertilizers increased all vegetative growth characters.

\begin{tabular}{|c|c|c|c|c|c|c|c|c|c|c|c|c|c|c|}
\hline \multirow[t]{2}{*}{ Treatments } & \multicolumn{2}{|c|}{$\begin{array}{l}\text { Plant length } \\
\text { (cm) }\end{array}$} & \multicolumn{2}{|c|}{$\begin{array}{c}\text { Stem diame- } \\
\text { ter }(\mathrm{cm})\end{array}$} & \multicolumn{2}{|c|}{$\begin{array}{l}\text { No. of leaves/ } \\
\text { Plant }\end{array}$} & \multicolumn{2}{|c|}{$\begin{array}{c}\text { No. of. brunches/ } \\
\text { plant }\end{array}$} & \multicolumn{2}{|c|}{$\begin{array}{c}\text { Dry weight } \\
\text { (g/plant) }\end{array}$} & \multicolumn{2}{|c|}{ Leaf area $\left(\mathrm{cm}^{2}\right)$} & \multicolumn{2}{|c|}{$\begin{array}{c}\text { leaf chloro- } \\
\text { phyll SPAD }\end{array}$} \\
\hline & 2018 & 2019 & 2018 & 2019 & 2018 & 2019 & 2018 & 2019 & 2018 & 2019 & 2018 & 2019 & 2018 & 2019 \\
\hline \multicolumn{15}{|c|}{ Fertilization levels } \\
\hline $100 \%$ (cont) & 44.83 & 43.04 & 1.28 & 1.25 & 183.69 & 170.21 & 24.26 & 22.91 & 43.51 & 40.79 & 451.13 & 423.99 & 79.44 & 76.86 \\
\hline $75 \%$ & 45.28 & 43.03 & 1.33 & 1.29 & 176.13 & 166.66 & 22.83 & 21.33 & 42.48 & 41.23 & 433.65 & 414.10 & 78.29 & 76.12 \\
\hline $50 \%$ & 45.01 & 42.94 & 1.29 & 1.23 & 172.65 & 168.17 & 21.73 & 20.34 & 40.01 & 38.54 & 423.49 & 399.59 & 78.01 & 75.17 \\
\hline $\begin{array}{l}\text { L.S.D at } 5 \% \\
\text { level }\end{array}$ & N.S & N.S & N.S & N.S & 5.17 & N.S & 0.06 & 0.98 & 0.98 & 1.18 & 1.07 & 14.22 & 0.33 & 0.98 \\
\hline \multicolumn{15}{|c|}{ Soil adding } \\
\hline Control & 41.21 & 37.94 & 1.19 & 1.20 & 127.65 & 121.99 & 19.75 & 17.92 & 31.85 & 30.44 & 331.64 & 312.86 & 74.34 & 72.33 \\
\hline Bio-fertilizer & 43.33 & 41.83 & 1.27 & 1.21 & 148.17 & 141.79 & 19.74 & 18.54 & 35.34 & 34.69 & 356.48 & 335.68 & 76.52 & 75.34 \\
\hline Compost & 44.33 & 42.50 & 1.27 & 1.23 & 173.22 & 157.15 & 20.78 & 20.11 & 41.94 & 39.32 & 387.73 & 359.83 & 79.83 & 75.42 \\
\hline Biochar & 45.47 & 43.50 & 1.33 & 1.31 & 180.03 & 178.00 & 24.33 & 23.59 & 43.13 & 41.06 & 482.19 & 461.80 & 79.62 & 76.12 \\
\hline $\begin{array}{l}\text { Bio-fertilizer+ } \\
\text { compost }\end{array}$ & 44.00 & 42.00 & 1.25 & 1.20 & 174.92 & 163.83 & 21.63 & 19.95 & 43.17 & 41.12 & 460.45 & 415.94 & 76.78 & 74.57 \\
\hline
\end{tabular}




\begin{tabular}{|c|c|c|c|c|c|c|c|c|c|c|c|c|c|c|}
\hline $\begin{array}{l}\text { Bio-fertilizer+ } \\
\text { biochar }\end{array}$ & 46.58 & 44.17 & 1.37 & 1.30 & 201.83 & 192.33 & 24.42 & 22.75 & 45.30 & 43.82 & 463.21 & 442.07 & 81.00 & 78.43 \\
\hline $\begin{array}{l}\text { Compost+ } \\
\text { biochar }\end{array}$ & 48.22 & 46.58 & 1.35 & 1.29 & 201.78 & 194.88 & 26.23 & 24.17 & 47.23 & 44.44 & 500.96 & 479.50 & 79.58 & 77.35 \\
\hline $\begin{array}{l}\text { Bio-fertiliz- } \\
\text { er+Compost+ } \\
\text { biochar }\end{array}$ & 47.17 & 45.50 & 1.37 & 1.33 & 212.33 & 196.79 & 26.66 & 25.16 & 48.05 & 46.59 & 506.03 & 492.78 & 80.97 & 78.83 \\
\hline $\begin{array}{l}\text { L.S.D at } 5 \% \\
\text { level }\end{array}$ & 0.77 & 0.95 & 0.09 & 0.06 & 5.56 & 5.70 & 0.89 & 0.80 & 0.57 & 0.82 & 5.90 & 6.96 & 0.75 & 0.80 \\
\hline
\end{tabular}

Table 6: Effect of mineral fertilization levels and treatments from the residual effect of biochar and compost as well as bio-fertilizers on the vegetative growth and chlorophyll leaf concentration of sweet pepper plants during the two seasons of 2018 and 2019.

\section{Residual effect of biochar, compost and bio-fertilizer}

As shown in Table (6) The data illustrated that, residual effect of biochar and compost after application them in the previous experiment (pea plants) with and without bio-fertilizer (azotobacter and phosphorene) increased the growth attributes i. e.; plant length, stem diameter, number of branches and leaves / plant, leaves area/plant, dry weight/ plant and chlorophyll leaf concentration in both growing seasons comparing to the control treatment which gave the lowest values. Especially, the residual effect of biochar and compost together with addition bio-fertilizer (azotobacter and phosphorene) gave the best values. Lehmann et al., 2003 and Steiner et al., 2008 reported that biochar increase maintain retention nutrient especially nitrogen content Moreover, it is a carbon rich solid material, which increases soil fertility by increasing moisture by holding capacity and attracting beneficial fungi and bacteria[Lehmann and Rondon(2006) and Lehmann(2007)]. Also, residual biochar increased availability of nutrients in the soil Widowati et al., (2017). Moreover, the nutrient release from biochar was effective, especially in the first year after application Sadowska et al., (2020). Many investigators reported that adding compost and biochar together reduced mineral fertilizer by increasing nutrient use efficiency, soil fertility and plant growth [Glaser and Birk (2012), Liu et al.,(2012), Schulz and Glaser (2012) and Fischer and Glaser (2012)]. Moreover, Mensah and Frimpong (2018) reported that biochar and compost applied lonely or together increased organic carbon, available phosphorus, and mineral nitrogen. Additionally, they increased growth and yield of maize varieties.

\section{Effect of the interaction}

Results in Table (7) clear that, there were noticeable superior values of growth attributes and chlorophyll leaf concentration of leaves plant with mineral fertilizer additions plus the residual effect of biochar, compost and bio-fertilizer (azotobacter and phosphorene) treatments. Especially the treatments which fertilized by $100 \%$ then $75 \%$ of recommended fertilizer with residual effect of biochar, compost and bio-fertilizers these results were true in both growing seasons. The same trend was noticed by several investigators, Duku et al., (2011) which revealed that biochar adding lead to a reduction in mineral fertilizer. Similar results obtained by Lehmann et al., (2003), (Widowati et al., 2011) and Widowati et al., (2012) reported that biochar can maintain nutrient in the fertilizer to prevent leaching. Since, biochar can reduce adding $\mathrm{N}$ fertilizer up to70\%. This is because biochar can manage $\mathrm{N}$ release by urea fertilizer. Also, Libing Pan et al., (2020) reported that, water content (27.4-65.1\%), total carbon (25.4-53.6\%), available nutrients (N, P) and decreased bulk density (3.2-23.9\%). 
Effect of Biochar, Compost and bio-fertilizer on Pea Yield Then, Study its Residual Effect on the Subsequent Pepper Crop

\begin{tabular}{|c|c|c|c|c|c|c|c|c|c|c|c|c|c|c|c|}
\hline \multirow{2}{*}{\multicolumn{2}{|c|}{ Treatments }} & \multicolumn{2}{|c|}{$\begin{array}{c}\text { Plant length } \\
\text { (cm) }\end{array}$} & \multicolumn{2}{|c|}{$\begin{array}{c}\text { Stem diame- } \\
\operatorname{ter}(\mathrm{cm})\end{array}$} & \multicolumn{2}{|c|}{$\begin{array}{c}\text { No. of leaves/ } \\
\text { Plant }\end{array}$} & \multicolumn{2}{|c|}{$\begin{array}{c}\text { No. of. } \\
\text { brunches/ } \\
\text { plant }\end{array}$} & \multicolumn{2}{|c|}{$\begin{array}{c}\text { Dry weight } \\
\text { (g/plant) }\end{array}$} & \multicolumn{2}{|c|}{ Leaf area $\left(\mathrm{cm}^{2}\right)$} & \multicolumn{2}{|c|}{$\begin{array}{c}\text { leaf chloro- } \\
\text { phyll SPAD }\end{array}$} \\
\hline & & 2018 & 2019 & 2018 & 2019 & 2018 & 2019 & 2018 & 2019 & 2018 & 2019 & 2018 & 2019 & 2018 & 2019 \\
\hline \multirow{8}{*}{$\stackrel{\circ}{\circ}$} & Control & 42.00 & 37.33 & 1.25 & 1.20 & 129.50 & 121.50 & 20.14 & 18.20 & 34.30 & 33.20 & 342.75 & 314.06 & 71.90 & 70.50 \\
\hline & $\begin{array}{l}\text { Bio-fertil- } \\
\text { izer }\end{array}$ & 42.00 & 41.00 & 1.25 & 1.20 & 148.00 & 141.00 & 20.30 & 18.50 & 35.20 & 34.90 & 353.54 & 330.66 & 77.00 & 76.05 \\
\hline & Compost & 45.00 & 43.50 & 1.30 & 1.25 & 183.00 & 161.50 & 22.84 & 21.67 & 44.10 & 41.10 & 442.19 & 408.65 & 81.70 & 76.50 \\
\hline & Biochar & 45.00 & 43.00 & 1.30 & 1.30 & 184.00 & 182.00 & 25.00 & 24.33 & 45.40 & 41.88 & 493.55 & 450.44 & 75.80 & 74.85 \\
\hline & $\begin{array}{l}\text { Bio-fer- } \\
\text { tilizer+ } \\
\text { compost }\end{array}$ & 42.00 & 41.00 & 1.10 & 1.10 & 182.00 & 172.00 & 23.94 & 21.71 & 45.20 & 40.40 & 474.46 & 427.56 & 78.30 & 74.40 \\
\hline & $\begin{array}{l}\text { Bio-fer- } \\
\text { tilizer+ } \\
\text { biochar }\end{array}$ & 47.50 & 46.00 & 1.30 & 1.25 & 205.00 & 191.00 & 26.01 & 25.67 & 45.90 & 44.06 & 468.11 & 462.51 & 85.40 & 82.30 \\
\hline & $\begin{array}{l}\text { Compost+ } \\
\text { biochar }\end{array}$ & 48.17 & 46.48 & 1.34 & 1.27 & 209.00 & 194.67 & 27.83 & 26.42 & 48.70 & 44.08 & 517.72 & 492.24 & 79.40 & 78.70 \\
\hline & $\begin{array}{l}\text { Bio-fer- } \\
\text { tilizer+ } \\
\text { Compost+ } \\
\text { biochar }\end{array}$ & 47.00 & 46.00 & 1.40 & 1.40 & 229.00 & 198.00 & 28.00 & 26.75 & 49.30 & 46.70 & 516.69 & 505.76 & 86.00 & 81.55 \\
\hline \multirow{8}{*}{ เั้ } & Control & 42.25 & 40.50 & 1.20 & 1.25 & 127.81 & 121.16 & 20.00 & 18.00 & 32.86 & 30.31 & 332.95 & 312.74 & 76.40 & 74.50 \\
\hline & $\begin{array}{l}\text { Bio-fertil- } \\
\text { izer }\end{array}$ & 46.00 & 43.00 & 1.30 & 1.23 & 145.00 & 137.38 & 19.66 & 18.62 & 35.17 & 34.98 & 364.52 & 339.01 & 76.65 & 75.88 \\
\hline & Compost & 44.00 & 42.00 & 1.30 & 1.25 & 172.00 & 151.56 & 20.00 & 20.00 & 42.50 & 41.70 & 360.40 & 332.14 & 78.50 & 76.10 \\
\hline & Biochar & 45.00 & 43.00 & 1.40 & 1.35 & 176.50 & 175.00 & 24.99 & 23.94 & 42.25 & 41.47 & 478.01 & 476.66 & 83.40 & 77.65 \\
\hline & $\begin{array}{l}\text { Bio-fer- } \\
\text { tilizer+ } \\
\text { compost }\end{array}$ & 44.00 & 42.00 & 1.35 & 1.30 & 166.42 & 157.50 & 20.97 & 19.36 & 45.20 & 43.90 & 449.19 & 415.46 & 74.60 & 73.45 \\
\hline & $\begin{array}{l}\text { Bio-fer- } \\
\text { tilizer+ } \\
\text { biochar }\end{array}$ & 46.00 & 43.00 & 1.40 & 1.35 & 208.50 & 196.00 & 24.25 & 22.33 & 45.60 & 44.10 & 456.92 & 434.88 & 76.50 & 75.15 \\
\hline & $\begin{array}{l}\text { Compost+ } \\
\text { biochar }\end{array}$ & 47.00 & 45.25 & 1.30 & 1.30 & 201.33 & 196.30 & 25.92 & 23.30 & 47.90 & 45.36 & 509.65 & 494.51 & 81.60 & 78.45 \\
\hline & $\begin{array}{l}\text { Bio-fer- } \\
\text { tilizer+ } \\
\text { Compost+ } \\
\text { biochar }\end{array}$ & 48.00 & 45.50 & 1.40 & 1.35 & 211.50 & 198.38 & 26.88 & 25.06 & 48.35 & 47.98 & 517.53 & 507.43 & 78.70 & 77.80 \\
\hline
\end{tabular}


Effect of Biochar, Compost and bio-fertilizer on Pea Yield Then, Study its Residual Effect on the Subsequent Pepper Crop

\begin{tabular}{|c|c|c|c|c|c|c|c|c|c|c|c|c|c|c|c|}
\hline \multirow{8}{*}{ 今े } & Control & 39.39 & 36.00 & 1.13 & 1.15 & 125.65 & 123.30 & 19.10 & 17.55 & 28.40 & 27.80 & 319.22 & 311.77 & 74.73 & 72.00 \\
\hline & $\begin{array}{l}\text { Bio-fertil- } \\
\text { izer }\end{array}$ & 42.00 & 41.50 & 1.25 & 1.20 & 151.50 & 147.00 & 19.25 & 18.50 & 35.65 & 34.18 & 351.37 & 337.38 & 75.90 & 74.10 \\
\hline & Compost & 44.00 & 42.00 & 1.20 & 1.20 & 164.67 & 158.38 & 19.51 & 18.67 & 39.23 & 35.17 & 360.59 & 338.69 & 79.30 & 73.65 \\
\hline & Biochar & 46.40 & 44.50 & 1.30 & 1.27 & 179.58 & 177.00 & 23.00 & 22.50 & 41.73 & 39.82 & 475.01 & 458.31 & 79.65 & 75.85 \\
\hline & $\begin{array}{l}\text { Bio-fer- } \\
\text { tilizer+ } \\
\text { compost }\end{array}$ & 46.00 & 43.00 & 1.30 & 1.20 & 176.33 & 162.00 & 19.98 & 18.77 & 39.10 & 39.05 & 457.71 & 404.81 & 77.45 & 75.85 \\
\hline & $\begin{array}{l}\text { Bio-fer- } \\
\text { tilizer+ } \\
\text { biochar }\end{array}$ & 46.25 & 43.50 & 1.40 & 1.30 & 192.00 & 190.00 & 23.00 & 20.25 & 44.40 & 43.30 & 464.61 & 428.83 & 81.10 & 77.85 \\
\hline & $\begin{array}{l}\text { Compost+ } \\
\text { biochar }\end{array}$ & 49.50 & 48.00 & 1.40 & 1.30 & 195.00 & 193.67 & 24.93 & 22.80 & 45.10 & 43.88 & 475.50 & 451.75 & 77.75 & 74.90 \\
\hline & $\begin{array}{l}\text { Bio-fer- } \\
\text { tilizer+ } \\
\text { Compost+ } \\
\text { biochar }\end{array}$ & 46.50 & 45.00 & 1.30 & 1.25 & 196.50 & 194.00 & 25.09 & 23.67 & 46.50 & 45.10 & 483.88 & 465.15 & 78.20 & 77.15 \\
\hline \multicolumn{2}{|c|}{ L.S.D at $5 \%$ level } & 0.47 & 058 & N.S & N.S & 3.37 & N.S & 0.54 & N.S & 0.35 & 0.49 & 3.58 & 4.22 & 0.45 & 0.49 \\
\hline
\end{tabular}

Table 7: Effect of the interaction between mineral fertilization levels and the residual effect of biochar and compost as well as bio-fertilizers treatments on the vegetative growth and chlorophyll leaf concentration of sweet pepper plants during the two seasons of 2018 and 2019.

\section{Physical characters of fruits}

\section{Effect of mineral fertilizer levels}

Data in Table (8) clear that, the least amount of NPK (50\%) increases the average fruit length of the sweet pepper, on other contrary the addition of $100 \%$ of recommended mineral fertilizer led to a significant increase in the percentage of dry matter in fruits and fruit V.C concentration. Meanwhile the rates of NPK did not record significant effects between them on fruit diameter, flesh thickness and loculi number. These trend of results obtained in both seasons under investigation. Many investigators reported that increasing the rate of NPK-fertilizer increased fruit quality. These results are agreement with Gaafar et al., (2019) and El-Atbany and Byan (2019) on pepper.

\section{Residual effect of biochar, compost and bio-fertilizer}

Concerning the residual effect of biochar and compost after application those during first excrement data listed that, the residual effect of biochar or compost alone or interacted together bio-fertilizer (azotobacter and phosphorene) gave the best values of physical characters of fruits and V.C concentration except fruit diameter and the fruit flesh thickness which recorded no significance effect compared with non adding treatment (control) as secured in Table (8). Data also mentioned that the residual effect of adding biochar alone increased percentage of dry matter in fruits meanwhile it added with compost or bio fertilizer obtained the best values fruit length and loculi number. These results were noticed in the both growing seasons. Biochar used to maintain the ecological resilience by using forest and cattle wastes to increase crop production and improve soil fertility for long time period Saxena et al., (2017) also, they reported that mixing of bio -inoculants to biochar is useful practice to increase crop productivity. 
Effect of Biochar, Compost and bio-fertilizer on Pea Yield Then, Study its Residual Effect on the Subsequent Pepper Crop

\begin{tabular}{|c|c|c|c|c|c|c|c|c|c|c|c|c|}
\hline \multirow[t]{2}{*}{ Treatments } & \multicolumn{2}{|c|}{ Fruit length $(\mathrm{cm})$} & \multicolumn{2}{|c|}{$\begin{array}{l}\text { Fruit diame- } \\
\text { ter }(\mathrm{cm})\end{array}$} & \multicolumn{2}{|c|}{$\begin{array}{c}\text { Flesh thickness } \\
\text { (cm) }\end{array}$} & \multicolumn{2}{|c|}{ Number of loculi } & \multicolumn{2}{|c|}{ Dry matter \% } & \multicolumn{2}{|c|}{ Vit C $(\mathrm{mg} / 100 \mathrm{~g})$} \\
\hline & 2018 & 2019 & 2018 & 2019 & 2018 & 2019 & 2018 & 2019 & 2018 & 2019 & 2018 & 2019 \\
\hline \multicolumn{13}{|c|}{ Fertilizer rates } \\
\hline $100 \%$ (cont) & 6.78 & 6.50 & 6.41 & 6.16 & 0.47 & 0.45 & 3.81 & 3.70 & 8.94 & 8.78 & 189.66 & 191.74 \\
\hline $75 \%$ & 6.69 & 6.77 & 6.33 & 6.20 & 0.48 & 0.46 & 3.75 & 3.67 & 8.84 & 8.57 & 176.72 & 174.81 \\
\hline $50 \%$ & 6.98 & 6.81 & 6.26 & 6.10 & 0.47 & 0.46 & 3.74 & 3.66 & 8.82 & 8.56 & 182.75 & 180.10 \\
\hline L.S.D at $5 \%$ level & 0.11 & 0.02 & N.S & N.S & N.S & N.S & N.S & N.S & 0.04 & 0.11 & 4.57 & 5.62 \\
\hline \multicolumn{13}{|c|}{ Soil adding } \\
\hline Control & 6.45 & 6.29 & 5.91 & 5.87 & 0.44 & 0.47 & 3.49 & 3.39 & 7.49 & 7.31 & 167.12 & 175.94 \\
\hline Bio-fertilizer & 6.46 & 6.51 & 6.34 & 6.07 & 0.50 & 0.46 & 3.76 & 3.65 & 8.84 & 8.49 & 188.98 & 185.51 \\
\hline Compost & 6.28 & 6.38 & 6.34 & 6.15 & 0.46 & 0.44 & 3.78 & 3.63 & 9.09 & 9.01 & 189.08 & 186.50 \\
\hline Biochar & 7.11 & 6.86 & 6.20 & 6.31 & 0.47 & 0.44 & 3.80 & 3.73 & 9.38 & 9.19 & 189.47 & 187.53 \\
\hline $\begin{array}{l}\text { Bio-fertilizer+ } \\
\text { compost }\end{array}$ & 6.94 & 6.69 & 6.32 & 6.04 & 0.47 & 0.44 & 3.79 & 3.66 & 9.08 & 8.95 & 190.43 & 188.28 \\
\hline $\begin{array}{l}\text { Bio-fertilizer+ } \\
\text { biochar }\end{array}$ & 7.07 & 6.84 & 6.42 & 6.15 & 0.50 & 0.47 & 3.79 & 3.73 & 8.43 & 8.25 & 180.39 & 178.53 \\
\hline $\begin{array}{l}\text { Compost+ bio- } \\
\text { char }\end{array}$ & 7.08 & 6.91 & 6.48 & 6.21 & 0.46 & 0.45 & 3.87 & 3.78 & 9.35 & 8.90 & 176.84 & 174.94 \\
\hline $\begin{array}{l}\text { Bio-fertilizer+ } \\
\text { Compost+ bio- } \\
\text { char }\end{array}$ & 7.16 & 7.03 & 6.66 & 6.44 & 0.48 & 0.47 & 3.86 & 3.85 & 9.28 & 8.98 & 182.03 & 180.51 \\
\hline L.S.D at $5 \%$ level & 0.09 & 0.10 & N.S & N.S & N.S & N.S & 0.11 & 0.21 & 0.05 & 0.13 & 4.76 & 3.29 \\
\hline
\end{tabular}

Table 8: Effect of mineral fertilization levels and treatments of the residual effect by biochar and compost as well as bio-fertilizers on some physical characters of sweet pepper fruits and V.C concentration during the two seasons of 2018 and 2019.

\section{Effect of the interaction}

Data in Table (9) registered that the average of fruit length and dry matter percent in fruits increased significantly in all interactions treatments between mineral fertilizer additions plus the residual effect of biochar, compost and bio-fertilizer (azotobacter and phosphorene) treatments, comparing to mineral fertilizer additions alone. Especially, the plants fertilized with any rate of mineral fertilizers plus residual effect of biochar or compost with or without bio-fertilizers treatments. Regarding the average of fruit diameter, the fruit flesh thickness and fruit loculi number results cleared that the differences did not reach to significant level at $5 \%$ in both seasons. These results may be due to that, using organic fertilizers and biochar increases nutrient stocks, decreases nutrient leaching, biochar allows better nutrients uptake which reflects for producing superior plants and increases crop productivity Steiner et al., (2007). On the other side, adding biochar with nitrogen fertilization appears to be promising practice to improve sustainability of intensive agriculture by enhancing properties of soil Horák et al., (2020) and Libing Pan et al., (2020). Some studies reported that mixed compost with biochar encourage soil fertility by increasing nutrient contents, improving soil structure, and water retention capacity thus reduce fertilizer application and increase plant growth [Glaser and Birk (2012), Liu et al., (2012), Schulz and Glaser (2012) and Fischer and Glaser (2012)]. 
Effect of Biochar, Compost and bio-fertilizer on Pea Yield Then, Study its Residual Effect on the Subsequent Pepper Crop

\begin{tabular}{|c|c|c|c|c|c|c|c|c|c|c|c|c|c|}
\hline \multirow{2}{*}{\multicolumn{2}{|c|}{ Treatments }} & \multicolumn{2}{|c|}{$\begin{array}{c}\text { Fruit length } \\
\text { (cm) }\end{array}$} & \multicolumn{2}{|c|}{$\begin{array}{c}\text { Fruit diameter } \\
(\mathrm{cm})\end{array}$} & \multicolumn{2}{|c|}{$\begin{array}{c}\text { Flesh thickness } \\
\text { (cm) }\end{array}$} & \multicolumn{2}{|c|}{$\begin{array}{c}\text { Number of } \\
\text { loculi }\end{array}$} & \multicolumn{2}{|c|}{ Dry matter \% } & \multicolumn{2}{|c|}{ Vit C $(\mathrm{mg} / 100 \mathrm{~g})$} \\
\hline & & 2018 & 2019 & 2018 & 2019 & 2018 & 2019 & 2018 & 2019 & 2018 & 2019 & 2018 & 2019 \\
\hline \multirow{8}{*}{ ஓి } & Control & 6.50 & 6.25 & 6.05 & 5.89 & 0.45 & 0.40 & 3.50 & 3.35 & 7.98 & 7.61 & 162.40 & 195.50 \\
\hline & Bio-fertilizer & 6.88 & 6.50 & 6.49 & 6.14 & 0.53 & 0.50 & 3.75 & 3.71 & 8.71 & 8.30 & 208.80 & 203.46 \\
\hline & Compost & 6.50 & 6.30 & 6.53 & 6.40 & 0.45 & 0.45 & 3.83 & 3.62 & 9.19 & 9.57 & 205.90 & 203.00 \\
\hline & Biochar & 6.90 & 6.40 & 6.30 & 6.17 & 0.42 & 0.40 & 3.86 & 3.78 & 9.38 & 9.24 & 185.60 & 183.28 \\
\hline & $\begin{array}{l}\text { Bio-fertilizer+ } \\
\text { compost }\end{array}$ & 6.79 & 6.53 & 6.20 & 5.90 & 0.43 & 0.42 & 3.84 & 3.67 & 9.30 & 9.48 & 207.06 & 205.32 \\
\hline & $\begin{array}{l}\text { Bio-fertilizer+ } \\
\text { biochar }\end{array}$ & 6.80 & 6.57 & 6.34 & 5.92 & 0.50 & 0.48 & 3.86 & 3.75 & 8.11 & 8.03 & 185.60 & 184.33 \\
\hline & \begin{tabular}{|l} 
Compost+ \\
biochar
\end{tabular} & 6.90 & 6.70 & 6.50 & 6.28 & 0.45 & 0.43 & 3.93 & 3.85 & 9.65 & 9.09 & 180.96 & 179.22 \\
\hline & $\begin{array}{l}\text { Bio-fertilizer+ } \\
\text { Compost+ } \\
\text { biochar }\end{array}$ & 7.00 & 6.75 & 6.90 & 6.60 & 0.52 & 0.50 & 3.90 & 3.89 & 9.19 & 8.95 & 180.96 & 179.80 \\
\hline \multirow{8}{*}{ है } & Control & 6.55 & 6.50 & 5.96 & 6.14 & 0.45 & 0.50 & 3.47 & 3.50 & 7.30 & 7.29 & 169.00 & 167.32 \\
\hline & Bio-fertilizer & 5.66 & 6.65 & 6.20 & 6.05 & 0.50 & 0.45 & 3.77 & 3.61 & 8.96 & 8.55 & 170.23 & 167.48 \\
\hline & Compost & 5.79 & 6.45 & 6.30 & 6.13 & 0.48 & 0.43 & 3.74 & 3.63 & 9.10 & 8.77 & 175.74 & 173.23 \\
\hline & Biochar & 7.15 & 6.94 & 6.13 & 6.16 & 0.48 & 0.47 & 3.83 & 3.67 & 9.42 & 9.33 & 185.60 & 184.44 \\
\hline & $\begin{array}{l}\text { Bio-fertilizer+ } \\
\text { compost }\end{array}$ & 7.00 & 6.78 & 6.49 & 6.39 & 0.50 & 0.45 & 3.77 & 3.63 & 8.75 & 8.37 & 176.32 & 175.07 \\
\hline & $\begin{array}{l}\text { Bio-fertilizer+ } \\
\text { biochar }\end{array}$ & 7.20 & 6.90 & 6.60 & 6.36 & 0.50 & 0.46 & 3.77 & 3.77 & 8.41 & 8.24 & 180.98 & 177.49 \\
\hline & \begin{tabular}{|l} 
Compost+ \\
biochar
\end{tabular} & 7.00 & 6.82 & 6.43 & 6.03 & 0.45 & 0.45 & 3.84 & 3.74 & 9.40 & 8.96 & 174.00 & 173.29 \\
\hline & $\begin{array}{l}\text { Bio-fertilizer+ } \\
\text { Compost+ } \\
\text { biochar } \\
\end{array}$ & 7.19 & 7.08 & 6.55 & 6.35 & 0.45 & 0.43 & 3.84 & 3.82 & 9.40 & 9.01 & 181.85 & 180.19 \\
\hline \multirow{8}{*}{ ถి } & Control & 6.30 & 6.13 & 5.73 & 5.57 & 0.43 & 0.50 & 3.50 & 3.33 & 7.21 & 7.02 & 169.95 & 165.01 \\
\hline & Bio-fertilizer & 6.83 & 6.37 & 6.33 & 6.03 & 0.48 & 0.43 & 3.77 & 3.63 & 8.84 & 8.63 & 187.92 & 185.60 \\
\hline & Compost & 6.55 & 6.40 & 6.20 & 5.93 & 0.45 & 0.43 & 3.77 & 3.63 & 8.98 & 8.70 & 185.60 & 183.28 \\
\hline & Biochar & 7.27 & 7.23 & 6.16 & 6.60 & 0.50 & 0.45 & 3.73 & 3.73 & 9.35 & 8.99 & 197.20 & 194.88 \\
\hline & $\begin{array}{l}\text { Bio-fertilizer+ } \\
\text { compost }\end{array}$ & 7.03 & 6.78 & 6.26 & 5.82 & 0.47 & 0.45 & 3.76 & 3.67 & 9.18 & 9.00 & 187.92 & 184.44 \\
\hline & \begin{tabular}{|l} 
Bio-fertilizer+ \\
biochar
\end{tabular} & 7.20 & 7.06 & 6.33 & 6.17 & 0.50 & 0.48 & 3.75 & 3.67 & 8.77 & 8.48 & 174.59 & 173.76 \\
\hline & $\begin{array}{l}\text { Compost+ } \\
\text { biochar }\end{array}$ & 7.35 & 7.21 & 6.50 & 6.33 & 0.48 & 0.47 & 3.84 & 3.74 & 9.00 & 8.65 & 175.55 & 172.32 \\
\hline & $\begin{array}{l}\text { Bio-fertilizer+ } \\
\text { Compost+ } \\
\text { biochar }\end{array}$ & 7.30 & 7.27 & 6.53 & 6.38 & 0.48 & 0.48 & 3.83 & 3.84 & 9.25 & 8.98 & 183.28 & 181.54 \\
\hline \multicolumn{2}{|c|}{ L.S.D at $5 \%$ level } & 0.06 & 0.05 & N.S & N.S & N.S & N.S & N.S & N.S & 0.03 & 0.08 & 2.89 & 1.99 \\
\hline
\end{tabular}

Table 9: Effect of the interaction between mineral fertilization levels and the residual effect of biochar and compost as well as bio-fertilizers treatments on some physical characters of sweet pepper fruits and V.C concentration during the two seasons of 2018 and 2019. 


\section{Yield and its Components}

\section{Effect of fertilizer levels}

It is obvious in Table (10) that increasing levels of NPK mineral fertilizer from $50 \%$ to $100 \%$ from the recommended level of NPK gave significantly increase in average fruit weight, early and total fruit yield per fedden. But the highest number of fruit reproduced from plants grown in plods which fertilized by 50\% from the recommended level of NPK these results was true in both growing seasons. It can say that these treatments as apparent in Table (6) seemed obvious, increasing in plant growth which that reflect on yield and its components. The same tendency was given by Gaafar et al., (2019) and El-Atbany and Byan (2019) on pepper.

\section{Residual effect of biochar, compost and bio-fertilizer}

Regarding to the residual effect of biochar and compost after application those at (pea plants) with or without bio-fertilizers (azotobacter and phosphorene) results in Table (10) show that plants grown in plots of the pervious treatments significantly enhanced all the studied fruit parameters compared with control which gave the lowest values. Especially, the residual effect of the mixture of biochar, compost and bio-fertilizer (azotobacter and phosphorene) gave the best significant values in both growing seasons. This might be due to the biochar potency to increase maintain retention nutrient especially nitrogen content (Lehmann et al., 2003; Steiner et al., 2008). Moreover, using bio -inoculants with biochar is useful practice to enhance crop productivity Saxena et al., (2017). Also, Trupiano et al., (2017) and Mensah and Frimpong (2018) found that using biochar lonely or with compost enhance soil fertility and increase yield.

\begin{tabular}{|c|c|c|c|c|c|c|c|c|}
\hline \multirow[t]{2}{*}{ Treatments } & \multicolumn{2}{|c|}{ Average of fruit weight $(\mathrm{g})$} & \multicolumn{2}{|c|}{ Fruit number/plant } & \multicolumn{2}{|c|}{$\begin{array}{c}\text { Early yield- } \\
\text { Ton/fed }\end{array}$} & \multicolumn{2}{|c|}{$\begin{array}{c}\text { Total yield Ton/ } \\
\text { fed }\end{array}$} \\
\hline & 2018 & 2019 & 2018 & 2019 & 2018 & 2019 & 2018 & 2019 \\
\hline \multicolumn{9}{|c|}{ Fertilizer rates } \\
\hline $100 \%$ (cont) & 82.47 & 79.64 & 37.06 & 36.41 & 4.649 & 4.323 & 30.394 & 29.078 \\
\hline $75 \%$ & 80.37 & 78.11 & 39.61 & 38.53 & 4.470 & 4.277 & 29.871 & 28.817 \\
\hline $50 \%$ & 78.72 & 75.91 & 42.22 & 41.57 & 4.348 & 4.152 & 28.241 & 27.182 \\
\hline L.S.D at $5 \%$ level & 0.65 & 1.43 & 1.44 & 0.92 & 0.057 & 0.111 & 1.417 & 0.503 \\
\hline \multicolumn{9}{|c|}{ Soil adding } \\
\hline Control & 68.94 & 66.86 & 23.83 & 22.96 & 3.591 & 3.313 & 19.509 & 17.774 \\
\hline Bio-fertilizer & 78.27 & 74.48 & 42.55 & 41.74 & 4.392 & 4.179 & 25.975 & 25.291 \\
\hline Compost & 78.25 & 77.14 & 39.20 & 38.40 & 4.208 & 4.035 & 28.389 & 26.856 \\
\hline Biochar & 83.07 & 80.52 & 40.01 & 39.17 & 4.289 & 4.094 & 32.510 & 31.376 \\
\hline Bio-fertilizer+ compost & 80.78 & 77.37 & 41.87 & 41.29 & 4.428 & 4.205 & 30.627 & 29.587 \\
\hline Bio-fertilizer+ biochar & 81.68 & 79.07 & 41.88 & 41.04 & 4.817 & 4.458 & 31.876 & 30.832 \\
\hline Compost+ biochar & 83.01 & 80.07 & 43.07 & 42.47 & 5.081 & 4.797 & 32.688 & 31.897 \\
\hline Bio-fertilizer+ Compost+ biochar & 90.16 & 87.58 & 44.61 & 43.63 & 5.105 & 4.923 & 34.445 & 33.258 \\
\hline L.S.D at $5 \%$ level & 0.93 & 0.76 & 1.40 & 0.96 & 0.100 & 0.117 & 0.853 & 0.920 \\
\hline
\end{tabular}

Table 10: Effect of mineral fertilization levels and treatments of the residual effect by biochar and compost as well as bio-fertilizers on the total yield and its components of sweet pepper fruits during the two seasons of 2018 and 2019. 


\section{Effect of the interaction}

As seemed in Table (11) the statistical analysis of the data indicated that differences of interaction between fertilizer levels and residual effect of biochar, compost and bio-fertilizers (azotobacter and phosphorene) treatments on yield and its components were significant $5 \%$ level. Data clear that the superior values of average of fruit weight, average of fruit number/plant, early yield and total per fedden were observed by fertilizer additions with at any rate of mineral fertilizers plus the residual effect of biochar, compost and bio-fertilizer treatments especially, with the highest level of mineral fertilizers $100 \%$ NPK except average of fruit number/plant gave the best values with 50\% NPK at the same treatment. This trend of results was true in the two seasons. In this regard Duku et al., (2011) revealed that using biochar reduce mineral fertilizers used by farmers. Biochar prevent nutrient leaching. Since, biochar can reduce nitrogen fertilizer up to70\% as reported by Lehmann et al., (2003), (Widowati et al., 2011) and Widowati et al,. (2012). Moreover, EL-Shimi and Byan (2015) reported that, using charcoal improved yield, and its quality of eggplant fruits under $50 \%$ from the recommended fertilization and this mean that decreasing the quantity of mineral fertilizers by $50 \%$ without any reduction effect on eggplant fruit yield. Furthermore, Yeboah et al., (2016) found that, Maize yield improved by using organic fertilizer with half of the recommended mineral fertilizer application and biochar $2.5 \mathrm{t} / \mathrm{ha}$. Widowati et al., (2017) showed that residual biochar lonely or with different levels of potassium application increased maize yield. Also, Horák et al., (2020) cleared that using biochar with $\mathrm{N}$ fertilizer increased organic carbon in the soil (from 8 to 79\%) also the ability of biochar to improve the grain yields over the monitored period from $1 \%$ up to $42 \%$, but this effect of biochar significantly decreased two years after its addition to the soil.

\begin{tabular}{|c|c|c|c|c|c|c|c|c|c|}
\hline & \multirow[t]{2}{*}{ Treatments } & \multicolumn{2}{|c|}{$\begin{array}{c}\text { Average of fruit weight } \\
(\mathrm{g})\end{array}$} & \multicolumn{2}{|c|}{ Fruit number/plant } & \multicolumn{2}{|c|}{ Early yield(Ton/fed) } & \multicolumn{2}{|c|}{ Total yield ( Ton/fed) } \\
\hline & & 2018 & 2019 & 2018 & 2019 & 2018 & 2019 & 2018 & 2019 \\
\hline \multirow{8}{*}{ §ิ } & Control & 72.75 & 70.50 & 24.72 & 23.91 & 3.966 & 3.559 & 22.772 & 20.304 \\
\hline & Bio-fertilizer & 80.91 & 76.00 & 44.07 & 43.69 & 4.435 & 4.145 & 28.277 & 27.547 \\
\hline & Compost & 82.15 & 81.30 & 36.29 & 35.76 & 4.418 & 4.101 & 29.453 & 28.585 \\
\hline & Biochar & 86.62 & 83.50 & 33.99 & 33.54 & 4.158 & 4.001 & 32.930 & 31.819 \\
\hline & $\begin{array}{l}\text { Bio-fertilizer+ } \\
\text { compost }\end{array}$ & 80.37 & 75.80 & 37.48 & 37.16 & 4.753 & 4.434 & 30.675 & 29.382 \\
\hline & $\begin{array}{l}\text { Bio-fertilizer+ } \\
\text { biochar }\end{array}$ & 80.50 & 79.80 & 39.80 & 38.93 & 5.031 & 4.519 & 31.990 & 30.240 \\
\hline & Compost+ biochar & 83.49 & 80.75 & 39.48 & 38.81 & 5.207 & 4.848 & 32.111 & 31.310 \\
\hline & $\begin{array}{l}\text { Bio-fertilizer+ } \\
\text { Compost+ biochar }\end{array}$ & 92.97 & 89.48 & 40.63 & 39.50 & 5.225 & 4.975 & 34.946 & 33.440 \\
\hline \multirow{8}{*}{ i̊ } & Control & 68.30 & 66.12 & 24.96 & 24.03 & 3.563 & 3.296 & 19.926 & 18.503 \\
\hline & Bio-fertilizer & 79.00 & 74.37 & 43.71 & 42.58 & 4.379 & 4.251 & 27.558 & 26.410 \\
\hline & Compost & 76.72 & 75.64 & 39.87 & 38.62 & 4.134 & 4.040 & 28.185 & 26.381 \\
\hline & Biochar & 82.86 & 81.14 & 39.81 & 37.83 & 4.398 & 4.153 & 32.309 & 31.256 \\
\hline & $\begin{array}{l}\text { Bio-fertilizer+ } \\
\text { compost }\end{array}$ & 79.13 & 76.73 & 42.78 & 41.93 & 4.322 & 4.205 & 30.702 & 29.443 \\
\hline & $\begin{array}{l}\text { Bio-fertilizer+ } \\
\text { biochar }\end{array}$ & 82.67 & 80.42 & 40.55 & 39.50 & 4.816 & 4.522 & 32.577 & 31.934 \\
\hline & Compost+ biochar & 81.98 & 80.28 & 41.62 & 40.89 & 5.064 & 4.818 & 33.253 & 32.906 \\
\hline & $\begin{array}{l}\text { Bio-fertilizer+ } \\
\text { Compost+ biochar }\end{array}$ & 92.27 & 90.19 & 43.57 & 42.86 & 5.085 & 4.934 & 34.462 & 33.701 \\
\hline
\end{tabular}


Effect of Biochar, Compost and bio-fertilizer on Pea Yield Then, Study its Residual Effect on the Subsequent Pepper Crop

\begin{tabular}{|c|c|c|c|c|c|c|c|c|c|}
\hline \multirow{8}{*}{ ถึ } & Control & 65.77 & 63.95 & 21.82 & 20.94 & 3.245 & 3.084 & 15.828 & 14.514 \\
\hline & Bio-fertilizer & 74.90 & 73.08 & 39.86 & 38.95 & 4.361 & 4.143 & 22.089 & 21.916 \\
\hline & Compost & 75.89 & 74.49 & 41.45 & 40.82 & 4.073 & 3.966 & 27.528 & 25.602 \\
\hline & Biochar & 79.74 & 76.93 & 46.22 & 46.15 & 4.311 & 4.127 & 32.290 & 31.054 \\
\hline & $\begin{array}{l}\text { Bio-fertilizer+ } \\
\text { compost }\end{array}$ & 82.83 & 79.58 & 45.34 & 44.78 & 4.210 & 3.976 & 30.504 & 29.937 \\
\hline & $\begin{array}{l}\text { Bio-fertilizer+ } \\
\text { biochar }\end{array}$ & 81.86 & 77.00 & 45.30 & 44.70 & 4.603 & 4.332 & 31.062 & 30.323 \\
\hline & Compost+ biochar & 83.55 & 79.17 & 48.10 & 47.70 & 4.972 & 4.727 & 32.701 & 31.475 \\
\hline & $\begin{array}{l}\text { Bio-fertilizer+ } \\
\text { Compost+ biochar }\end{array}$ & 85.23 & 83.08 & 49.64 & 48.53 & 5.006 & 4.859 & 33.927 & 32.632 \\
\hline \multicolumn{2}{|c|}{ L.S.D at $5 \%$ level } & 0.56 & 0.46 & 0.85 & 0.57 & 0.061 & 0.071 & 0.518 & 0.558 \\
\hline
\end{tabular}

Table 11: Effect of the interaction between mineral fertilization levels and the residual effect of biochar and compost as well as bio-fertilizers treatments on the total yield and its components of sweet pepper fruits during the two seasons of 2018 and 2019.

\section{References}

1. Amarakoon D., et al. "Iron-, zinc-, and magnesium-rich field peas (Pisum sativum L.) with naturally low phytic acid: a potential food-based solution to global micronutrient malnutrition". J. Food Compost. Anal 27.1 (2012): 8-13.

2. Duku HM, S Gu and EB Hagan. "Biochar production potentials in Ghana-a review". Renewable and Sustainable Energy Review 15 (2011): 3539-3551.

3. EL-Shimi-Nahed MM and AI Usrya-Byan. "Effect of adding charcoal as natural organic matter and fertilizer levels recommendation on the growth and yield of eggplant plants". Egypt. J. Appl. Sci 30.4 (2015): 107-125.

4. Feleafel MN and ZM Mirdad. "Influence of Organic Nitrogen on the Snap Bean Grown in Sandy Soil”. International Journal of Agriculture \& Biology 16.1 (2014): 65-72.

5. Fischer D and B Glaser. "Synergisms between compost and biochar for sustainable soil amelioration". Management of Organic Waste, S. Kumar and A. Bharti, Eds (2012): 168-198.

6. Gaafar-Mona S., et al. "Effect of foliar and soil application of some residuals of sugar cane products (molasses and vinasses) with mineral fertilizer levels on growth, yield and quality of sweet pepper". Menoufia J. of plant production 4.5 (2019): $353-373$.

7. Ganie NA, RB Solanki and FA Allie. "Effect of bio-fertilizers on growth and yield of garden pea (Pisum sativum L.)”. Asian. J. Hort 4.2 (2010): 507-509.

8. Glaser B and J Birk. "State of the scientific knowledge on properties and genesis of Anthropogenic Dark Earths in Central Amazonia (terra preta de 'indio)". Geochimica et Cosmochimica Acta 82 (2012): 39-51.

9. Helgi $O$ and SA Rolfe. The Physiology of Flowering Plants. 4th ed., Cambridge University Press, Cambridge UK (2005): 100-106.

10. Horák J, V Šimanský and E Aydin. "Benefits of biochar and its combination with nitrogen fertilization for soil quality and grain yields of barley, wheat and corn". Journal of Elementology 25.2 (2020): 443-458.

11. Jayasinghe HASL and ANR Weerawansha. "Effect of compost and different NPK levels on growth and yield of three tomato (Solanum lycopersicum) Varieties in Sri Lanka”. J. of Advanced Agricultural Technologies 5.2 (2018): 129-133.

12. Lehmann J., et al. "Nutrient availability and leaching in an archaeological Anthrosol and a Ferralsol of the Central Amazon basin: Fertilizer, manure and charcoal amendments". Plant and Soil 249 (2003): 343-357.

13. Lehmann J, J Gaunt and M Rondon. "Bio-char sequestration in terrestrial ecosystems-A review”. Mitigation. Adapt. Strat. Global Change 11 (2006): 403- 427.

14. Lehmann J and M Rondon. "Bio-char soil management on highly weathered soils in the humid tropics". Biological approaches to sustainable soil systems (Ed.: N. Uphoff), CRC Press, Boca Raton, FL (2006): 517-530.

15. Lehmann J. “Bio-energy in the black, Front”. Ecol. Environ 5 (2007): 381-387. 
16. Libing Pan., et al. "The potential for biochar application in rubber plantations in Xishuangbanna, Southwest China: a pot trial”. Springer (2020).

17. Liu J., et al. "Short-term effect of biochar and compost on soil fertility and water status of a Dystric Cambisol in NE Germany under field conditions". Journal of Plant Nutrition, Soil Science 175.5 (2012): 1-10.

18. Mensah AK and Frimpong KA. "Biochar and/or compost applications improve soil properties, growth, and yield of maize grown in acidic rainforest and coastal savannah soils in Ghana". International Journal of Agronomy (2018).

19. Mishra A, K Prasad and G. Rai. "Effect of bio-fertilizer inoculation on growth and yield of dwarf field pea (Pisum sativum L.) in conjunction with different doses of chemical fertilizers". Journal of Agronomy 9.4 (2010): 163-168.

20. Piñero MC., et al. "Changes in the salinity tolerance of sweet pepper plants as affected by nitrogen form and high $\mathrm{CO}_{2} \mathrm{concentra-}^{-}$ tion". J. Plant Physiol 200 (2016): 18-27.

21. Ranganna C. "Manual of analysis of fruit vegetable products". Tatame. Graw Hill publishing company limited New Delhi (2nd ed) (1979): 105-119.

22. Rodríguez-Ruiz M., et al. "Characterization of the galactono-1,4-lactone dehydrogenase from pepper fruits and its modulation in the ascorbate biosynthesis. Role of nitric oxide". Redox Biol 12 (2017): 171-181.

23. Sadowska U., et al. "Biochar and its E_ects on plant-soil macronutrient cycling during a three-year field trial on sandy soil with peppermint (Mentha piperita L.). Part I: yield and macro Element content in soil and plant biomass". Agronomy 10.1950 (2020): 1-20.

24. Salwa A El-Atbany and Usrya AI Byan. "Effect of using water hyacinth compost comparing with some organic fertilizers and mineral fertilizer levels on growth and yield of sweet pepper". J. Hort. Sci. \& Ornamen. Plants 11.3 (2019): 204-213.

25. Saxena J, G Rana and M. Pandey. "Impact of addition of biochar along with Bacillus sp. on growth and yield of French beans". Sci. Hort 162 (2013): 351-356.

26. Saxena J, J Rawat and R Kumar. "Conversion of biomass waste into biochar and the effect on mung bean crop production". CLEAN - Soil Air Water (2017).

27. Schulz H and B Glaser. "Effects of biochar compared to organic and inorganic fertilizers on soil quality and plant growth in a greenhouse experiment". Journal of Plant Nutrition and Soil Science 175.3 (2012): 410-422.

28. Snedecor GW and WG Cochran. Statistial Methoods, 7th Ed., The Iowa state Univ., Press, Ames., Iowa, USA (1980): 83-94.

29. Steiner C., et al. "Nitrogen retention and plant uptake on a highly weathered central Amazonian Ferraisol amended with compost and charcoal”. Journal of Plant Nutrition and Soil Science 171 (2008): 893-899.

30. Steiner C., et al. "Long term effects of manure, charcoal and mineral fertilization on crop production and fertility on a highly weathered Central Amazonian upland soil”. Plant and Soil 291 (2007): 275-290.

31. Tagore GS., et al. "Effect of Rhizobium and phosphate solubilizing bacterial inoculants on symbiotic traits, nodule legheamoglobin and yield of chickpea genotypes”. Inter. J. Agro 58 (2013): 16-27.

32. Toungos DM. "The effects of different levels of inorganic fertilizer (npk 15:15:15,) on growth and yield of sweet pepper (capsicum annum) in Mubi, Nigeria”. International J. Develop. Res 7.6 (2017): 13120-13124.

33. Trupiano D., et al. "The Effects of biochar and its combination with compost on lettuce (Lactuca sativa L.) growth, soil properties, and soil microbial activity and abundance". International Journal of Agronomy (2017).

34. Byan-Usrya AI and Nahed MMEL-Shimi. "Influence of using some natural and biotical materials on growth and quality of pea yield”. Annals. Agric. Sci. Moshtohor 52.1 (2014): 111-121.

35. Wallace DH and HM Munger. "Studies of the physiological basis for yield differences. 1. growth and analysis of six dry bean varieties". Crop Sci 5 (1965): 343-348.

36. Widowati Utomo WH., et al. "Effect of biochar on the release and loss of nitrogen from urea fertilization". Journal of Agriculture and Food Technology 1.7 (2011): 127-132.

37. Widowati Utomo WH., et al. "The effect of biochar on the growth and $\mathrm{N}$ fertilizer requirement of maize (Zea mays L.) in green house experiment". Journal of Agricultural Science 4.5 (2012): 255-262. 
38. Widowati A., et al. "Residual effect of potassium fertilizer and biochar on growth and yield of maize in the second season". J. Degrade. Min. Land Manage 4.4 (2017): 881-889.

39. Zaghloul RA., et al. "Improvement of growth and yield of pea plants using integrated fertilization management”. Universal Journal of Agricultural Research 3.4 (2015): 135-143.

Volume 2 Issue 3 March 2022

(c) All rights are reserved by Nahed MM El-Shimi., et al. 